\title{
Weight Changes in Type 2 Diabetes and Cancer Risk: A Latent Class Trajectory Model Study
}

\author{
Britt W. Jensen $^{a, b} \quad$ Charlotte Watson $^{b}$ Nophar Geifman ${ }^{c, d}$ Jennifer L. Baker ${ }^{a}$ \\ Ellena Badrick ${ }^{b}$ Andrew $G$. Renehan ${ }^{b}$ \\ ${ }^{a}$ Center for Clinical Research and Prevention, Bispebjerg and Frederiksberg Hospital, The Capital Region, \\ Copenhagen, Denmark; ${ }^{b}$ Division of Cancer Sciences, School of Medical Sciences, Faculty of Biology, Medicine \\ and Health, University of Manchester, Manchester, UK; ' ${ }^{C}$ Centre for Health Informatics, University of Manchester, \\ Manchester, UK; ${ }^{\text {d} F a c u l t y ~ o f ~ H e a l t h ~ a n d ~ M e d i c a l ~ S c i e n c e s, ~ U n i v e r s i t y ~ o f ~ S u r r e y, ~ G u i l d f o r d, ~ U K ~}$
}

\section{Keywords}

Body mass index $\cdot$ Latent classes $\cdot$ Diabetes $\cdot$ Cancer

\begin{abstract}
Introduction: Body mass index (BMI) is often elevated at type 2 diabetes (T2D) diagnosis. Using latent class trajectory modelling (LCTM) of BMI, we examined whether weight loss after diagnosis influenced cancer incidence and all-cause mortality. Methods: From 1995 to 2010, we identified 7,708 patients with T2D from the Salford Integrated Record database (UK) and linked to the cancer registry for information on obesity-related cancer (ORC), non-ORC; and all-cause mortality. Repeated BMIs were used to construct sex-specific latent class trajectories. Hazard ratios (HRs) and 95\% confidence intervals ( $\mathrm{Cls}$ ) were estimated using Cox regression models. Results: Four sex-specific BMI classes were identified; stable-overweight, stable-obese, obese-slightly-decreasing, and obese-steeply-decreasing; comprising 41\%, $45 \%, 13 \%$, and $1 \%$ of women, and $45 \%, 37 \%, 17 \%$, and $1 \%$ of men, respectively. In women, the stable-obese class had similar ORC risks as the obese-slightly-decreasing class, whereas the stable-overweight class had lower risks. In men, the obese-slightly-decreasing class had higher risks of ORC
\end{abstract}

karger@karger.com www.karger.com/ofa

Karger!"

GOPEN ACCESS
C 2021 The Author(s).

Published by S. Karger AG, Basel

This is an Open Access article licensed under the Creative Commons Attribution-NonCommercial-4.0 International License (CC BY-NC) (http://www.karger.com/Services/OpenAccessLicense), applicable to the online version of the article only. Usage and distribution for commercial purposes requires written permission.
$(\mathrm{HR}=1.86,95 \% \mathrm{Cl}: 1.05-3.32)$ than the stable-obese class, while the stable-overweight class had similar risks No associations were observed for non-ORC. Compared to the stable-obese class, women ( $\mathrm{HR}=1.60,95 \% \mathrm{Cl}: 0.99-2.58)$ and men ( $\mathrm{HR}=2.37,95 \% \mathrm{Cl}: 1.66-3.39)$ in the obese-slightly-decreasing class had elevated mortality. No associations were observed for the stable-overweight classes. Conclusion: Patients who lost weight after T2D diagnosis had higher risks for ORC (in men) and higher all-cause mortality (both genders) than patients with stable obesity.

(C) 2021 The Author(s)

Published by S. Karger AG, Basel

\section{Introduction}

Body fatness, commonly approximated by body mass index (BMI), is an established risk factor for type 2 diabetes (T2D) [1] and several cancer types [2]. The International Agency for Research on Cancer [3] listed 13 cancers where there is sufficient evidence for causal association between increased body fatness and cancer risk. These have been termed obesity-related cancers (ORCs). In parallel, major reports [4] and meta-analyses [5, 6] have concluded that individuals with T2D have an in-
Correspondence to:

Britt W. Jensen, britt.wang.jensen@ regionh.dk 
creased risk for developing ORCs, such as breast, colorectal, endometrial, liver, and pancreatic cancers, compared with individuals without T2D. It is unclear, whether T2D per se, mutual risk factors, like excess body fatness, or anti-diabetes treatments, might explain these observations [7].

At the time of a T2D diagnosis, $\mathrm{BMI}$ is typically elevated (mean BMI approximately $30 \mathrm{~kg} / \mathrm{m}^{2}$ [8]), and subsequent weight changes, might affect later cancer risk. Four studies [9-12] have evaluated associations between a "one-off" BMI measure (typically at cohort entry) and cancer incidence in patients with T2D, and broadly support the hypothesis of overweight leading to an increased cancer risk in patients with T2D. A proportion of patients with T2D lose weight (either intentionally or non-intentionally) after diagnosis, and this might reduce risk of diabetes-associated diseases and mortality, but evidence is inconsistent. For example, cardiovascular disease (CVD) is the most common diabetes complication, and while weight loss is associated with improvements in CVD risk factors in patients with T2D [13] and may lead to diabetes remission [14], findings from studies evaluating associations of weight loss with CVD are inconclusive [15-17]. Recently, the ADDITION trial of screen-detected T2D [18], with 10 years of follow-up, reported that moderate weight loss yielded long-term CVD reduction, though this conclusion was based on only 229 individuals. A UK study found that gaining weight after T2D was associated with lower mortality in patients with a normal weight at diagnosis and higher mortality in patients with obesity grade III at diagnosis [19]. To our knowledge, BMI trajectories after T2D diagnosis and cancer risk has not been evaluated.

Here, we sought to identify different BMI trajectories after T2D diagnosis using latent class trajectory modelling (LCTM) and examine their association with cancer risk and all-cause mortality. We hypothesised that this data-driven approach could identify a group of patients with a decreasing BMI trajectory post-T2D diagnosis and that this group of patients would have a lower ORC risk and mortality.

\section{Methods}

The study was based on the Salford Integrated Record, a National Health Service database [8] established in 2007 including 248,913 patients from Salford city, UK [20]. Data are based on primary and secondary care records, obtained from 45 general practitioners (GPs) and one hospital [21].

We identified 12,221 patients in the cohort diagnosed with diabetes between January 1, 1995, and December 31, 2010, using
Read codes that describe a variety of information (including diagnoses and medication) on patients attending GPs (online suppl. Fig. S1; see www.karger.com/doi/10.1159/000520200 for all online suppl. material). We used a modified version of the method suggested by de Lusignan et al. [22] to identify patients with T2D: we searched for Read codes beginning with "C10," having "diabet" in the medical definition or an insulin prescription [8]. Individuals with gestational diabetes or type 1 diabetes ("Type-1" in diagnostic code, "C10F\%" in medical code, $<35$ years at diagnosis and at first prescribed insulin, and insulin prescribed $<6$ months after diagnosis) were excluded. Moreover, we excluded patients with only one text entry of T2D, and patients $>85$ years at diagnosis.

\section{Exposure Variables}

Information on BMI was obtained from the medical records. LCTM including all available BMI values from the date of T2D diagnosis (+/- one year) until ten years after diagnosis (or until a cancer diagnosis) were used to identify BMI trajectories. BMI values $<15$ and $>60 \mathrm{~kg} / \mathrm{m}^{2}$ were excluded as outliers. BMI values changing $>5$ BMI units in $<3$ months were excluded as measurement errors. If a patient had multiple BMI values in one day, a random value was chosen. Patients were categorized as under- or normal weight (BMI: $<25 \mathrm{~kg} / \mathrm{m}^{2}$ ), overweight (BMI: $25-29.9 \mathrm{~kg}$ / $\mathrm{m}^{2}$ ), obese I (BMI: $30-34.9 \mathrm{~kg} / \mathrm{m}^{2}$ ) or obese II or III (BMI: $>35 \mathrm{~kg} /$ $\mathrm{m}^{2}$ ) based on BMI at T2D diagnosis.

Information on smoking status (ever/never), anti-diabetes medication, $\mathrm{HbAlc}$, blood pressure, and cholesterol as close to the date of diagnosis with T2D as possible and within $+/-$ one year were available from the GP records. HbAlc values reported as percentages (Diabetes Complications and Control Trial method) were transformed into $\mathrm{mmol} / \mathrm{mol}$ according to the method suggested by the International Federation of Clinical Chemistry [23]. Deprivation score was derived using the Index of Multiple Deprivation (IMD) [24], and its distribution divided into quintiles from least deprived $(\mathrm{IMD}=1)$ to most deprived $(\mathrm{IMD}=5)$.

\section{Outcome Variables}

Incident cancers diagnosed between January 1, 1995, and December 31, 2010, were identified using the International Classification of Disease (ICD) version 10 codes as the first cancer by linkage with the National Cancer Intelligence Services, UK. ORCs were defined according to the 13 cancer forms identified as obesity-related according the International Agency for Research on Cancer; these include: breast, colon, corpus uteri, gallbladder, gastric cardia, kidney, liver meningioma, multiple myeloma, oesophagus, ovary, pancreas, rectal, and thyroid (online suppl. Table S1). NonORCs were defined as all cancers not considered obesity-related (including non-melanoma skin cancer). Information on date of death was available from the GP records.

\section{Generation of BMI Trajectories Using Latent Class Mixed}

Models

Using the Latent Class Mixed Models package and the "hlme" function in $\mathrm{R}$ (version 3.4.2), latent class trajectories were identified. Sex-specific models including one to seven classes were tested with linear, quadratic, and cubic slopes as a function of time (years) since T2D diagnosis. To account for individual variation around the mean trajectory, random effects modelled as a random intercept and a cubic slope were included. 
Fig. 1. BMI latent class trajectories for women and men. In the obese-steeply-decreasing class, BMI values were only available for a maximum of 7.8 and 8.5 years of follow-up for women and men with a mean of 4.5 and 4.9 years, respectively. BMI, body mass index.

\section{Men}

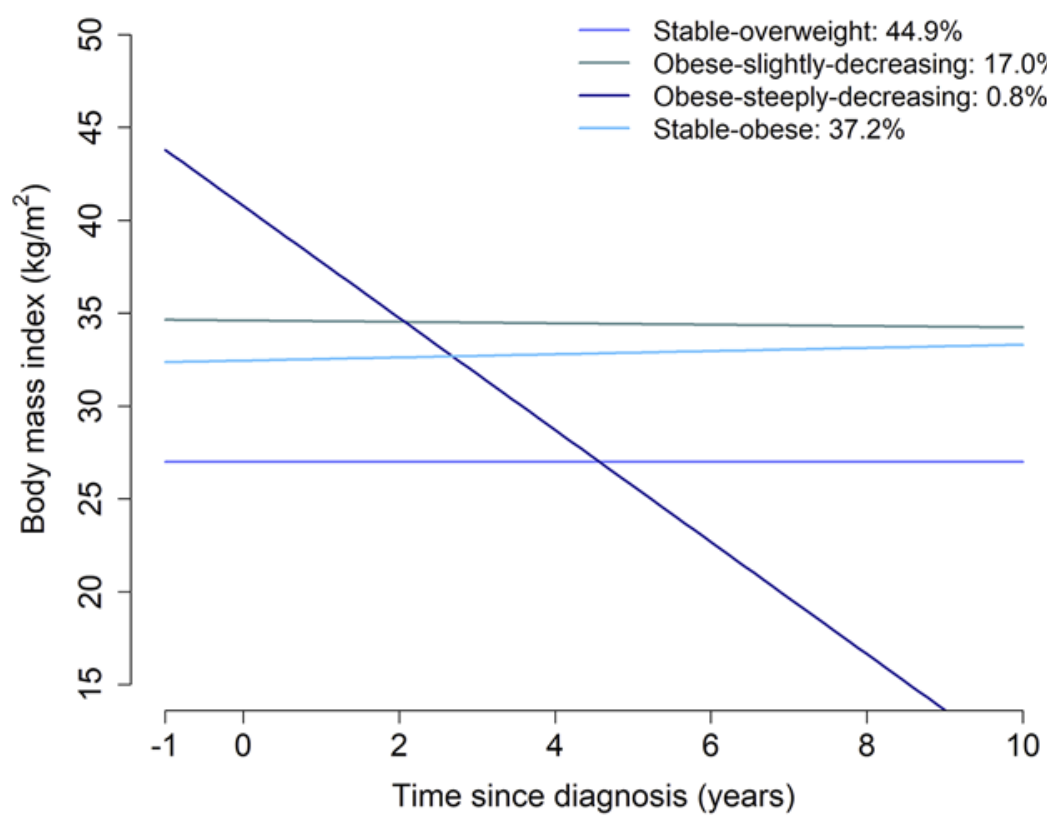

Women

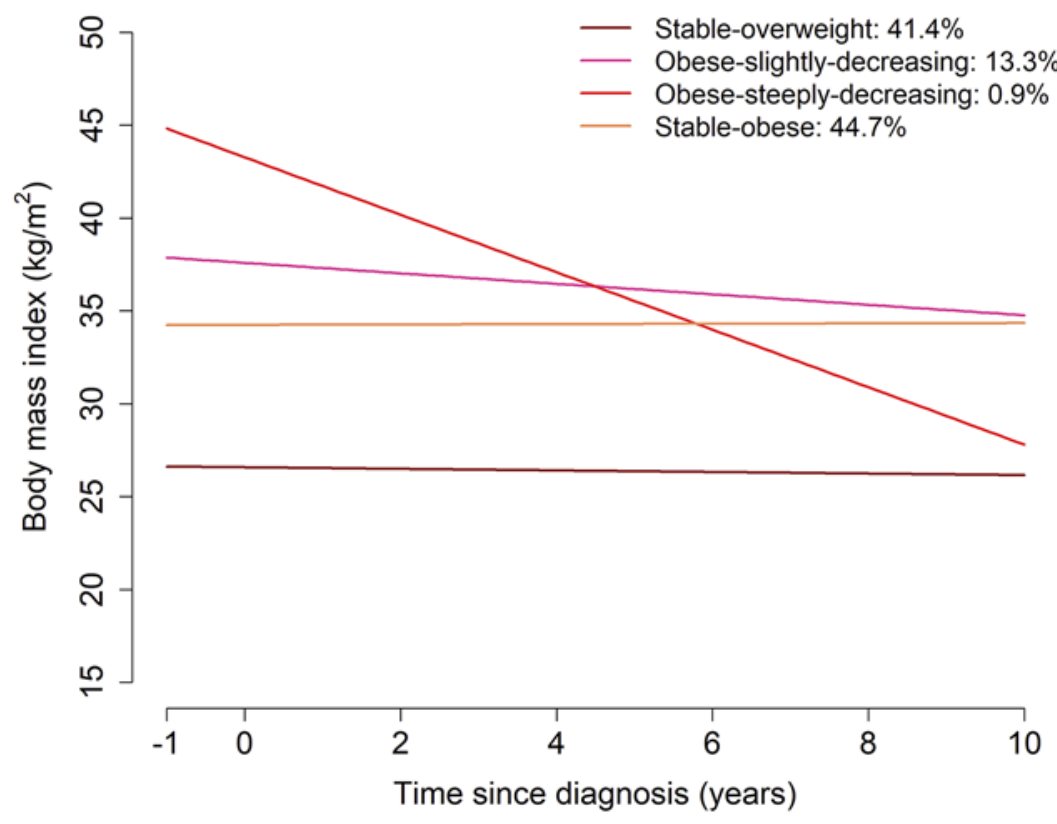

A step-wise approach, including: evaluation of the structure of the random effects and identification of the optimal number of classes based on a number of parameters, was used to identify the optimal model (online suppl. Fig. S2) [25]. A linear model with four classes was identified as the best model for women and men. To secure that the models converged at the global maximum, the models were repeated with multiple starting points $(n$
=20) using the "gridsearch" function in the Latent Class Mixed Model package.

The patients were assigned to the class with the highest posterior probability (online suppl. Fig. S3), and Figure 1 shows the mean estimated trajectory for each class throughout the study period. The trajectory models were repeated including only patients with a posterior probability $\geq 70 \%$, showing similar shapes 
Fig. 2. Chord plot illustrating the patients in each BMI latent class trajectory (below the horizontal line) and their categorization according to the WHO BMI categories (above the horizontal line) for women and men. BMI, body mass index.

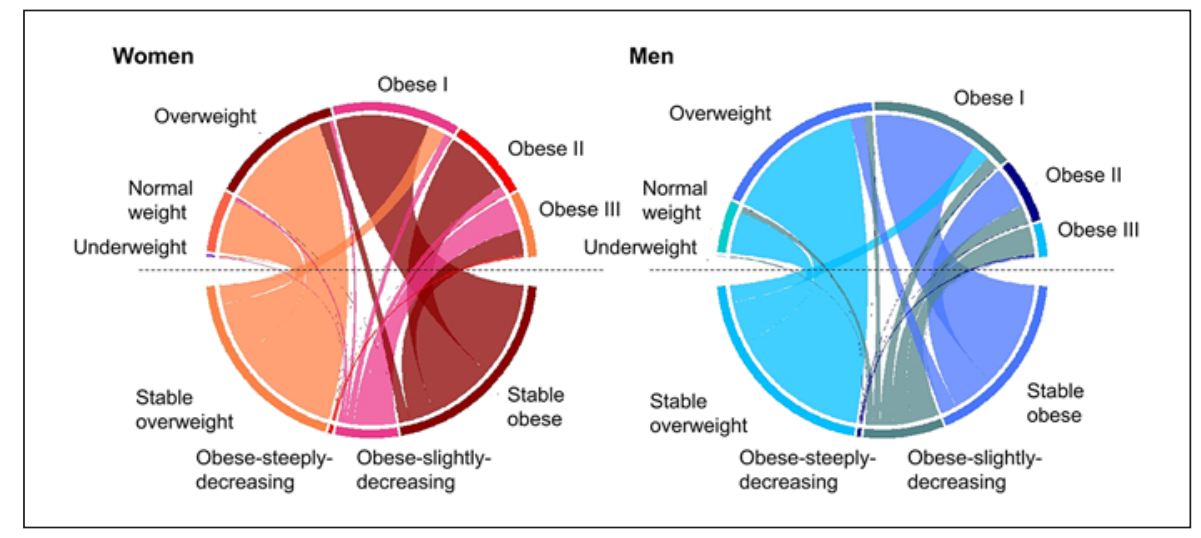

of the trajectories as the models including all patients (online suppl. Fig. S4).

\section{Statistical Analyses}

Differences in baseline characteristics among the four classes were tested using the $\chi^{2}$ test and ANOVA as appropriate. Chord plots were used to illustrate how patients were grouped based on $\mathrm{BMI}$ at T2D diagnosis (using the WHO BMI categories) compared to the identified BMI classes (Fig. 2). In the violin plots, the sexspecific distribution of age and BMI were presented for each class (online suppl. Fig. S5). Observation time was calculated as time to censoring.

Cox proportional hazards regression models were used to examine the associations between the identified BMI latent class trajectories (included as a categorical variable) and ORC, non-ORC and all-cause mortality with time since diagnosis as the time scale. Patients were followed from the date of T2D diagnosis until the date of a cancer diagnosis (in analyses of cancer), death or December 31, 2010, whichever came first. All analyses were conducted separately by sex. Due to the few patients and cancer cases in the obese-steeply-decreasing class, the results for this class are not reported.

The analyses were conducted in two models: (1) adjusted for age and year of T2D diagnosis and (2) additionally adjusted for smoking and deprivation area. Medication usage was not included in the Cox regression models as modelling a time varying exposure to drugs was not feasible given the limited sample size. In all analyses, the stable-obese class served as the reference as this class reflects the general BMI level at diagnosis with T2D and since we aimed to examine if a decreasing BMI trajectory was associated with lower ORC risk. The assumption of linearity was tested for the continuous co-variables by including a quadratic term of the variable. Age at diagnosis was significantly non-linearly associated with non-ORC and all-cause mortality among men $(p<0.03)$ and was included as a quadratic term in all models for both women and men.

No interactions were found between the BMI classes and covariables (age, year at diagnosis with T2D, smoking and deprivation area) when tested by inclusion of the product term of the exposure and the co-variable using the likelihood ratio test. The assumption of proportional hazards was tested by dividing the follow-up time as $0-2$ years and $>2$ years for ORC and non-ORC and divided as $0-5$ years and $>5$ years for all-cause mortality.

Latent BMI Classes, Diabetes, and Cancer
The Cox regression models were repeated including only patients with a posterior probability $>70 \%$. Associations with nonORC in men were examined excluding prostate cancer as it has been suggested to be obesity-related [26]. Further, we examined the associations between $\mathrm{BMI}$ at T2D diagnosis (categorized according to the WHO BMI categorization) and ORC, non-ORC, and all-cause mortality. The Obese I group was used as the reference to make the results comparable with those from the analyses on BMI latent class trajectories. Finally, analyses were adjusted for BMI at diagnosis with T2D to account for the variation in BMI within and between the classes.

\section{Results}

A total of 7,708 patients (3,344 women) were included in the analyses, with a median of $17 \mathrm{BMI}$ values per person (range 1-139). Patients excluded due to not having a BMI +/-1 year of T2D diagnosis were born and diagnosed with T2D earlier than patients included in the study. They were also younger at diagnosis and less likely to be smokers, while no sex-difference was observed (online suppl. Table S2). We identified four BMI latent class trajectories; "stable-overweight" (women: 41\%, men: 45\%; Fig. 1); "stable-obese" (women: 45\%, men: 37\%); "obese-slightly-decreasing" (women: 13\%, mean decrease in 10 years: $2.8 \mathrm{~kg} / \mathrm{m}^{2}$, men: $17 \%$, mean decrease in 10 years: $0.4 \mathrm{~kg} / \mathrm{m}^{2}$ ); and "obese-steeply-decreasing" (women: $1 \%$, men: $1 \%$ ).

Patients in the stable-overweight class were born earlier, were older at T2D diagnosis (Table 1; online suppl. Fig. S5) and were less likely to be prescribed Metformin. Men in the stable-obese class were less likely to be prescribed Sulphonylureas and women in the obese-steeplydecreasing and obese-slightly-decreasing classes had more BMI values available than the other classes. No statistically significant differences were observed in the pro- 
Table 1. Characteristics of the identified BMI latent class trajectories in women and men, SIRs ${ }^{1}$

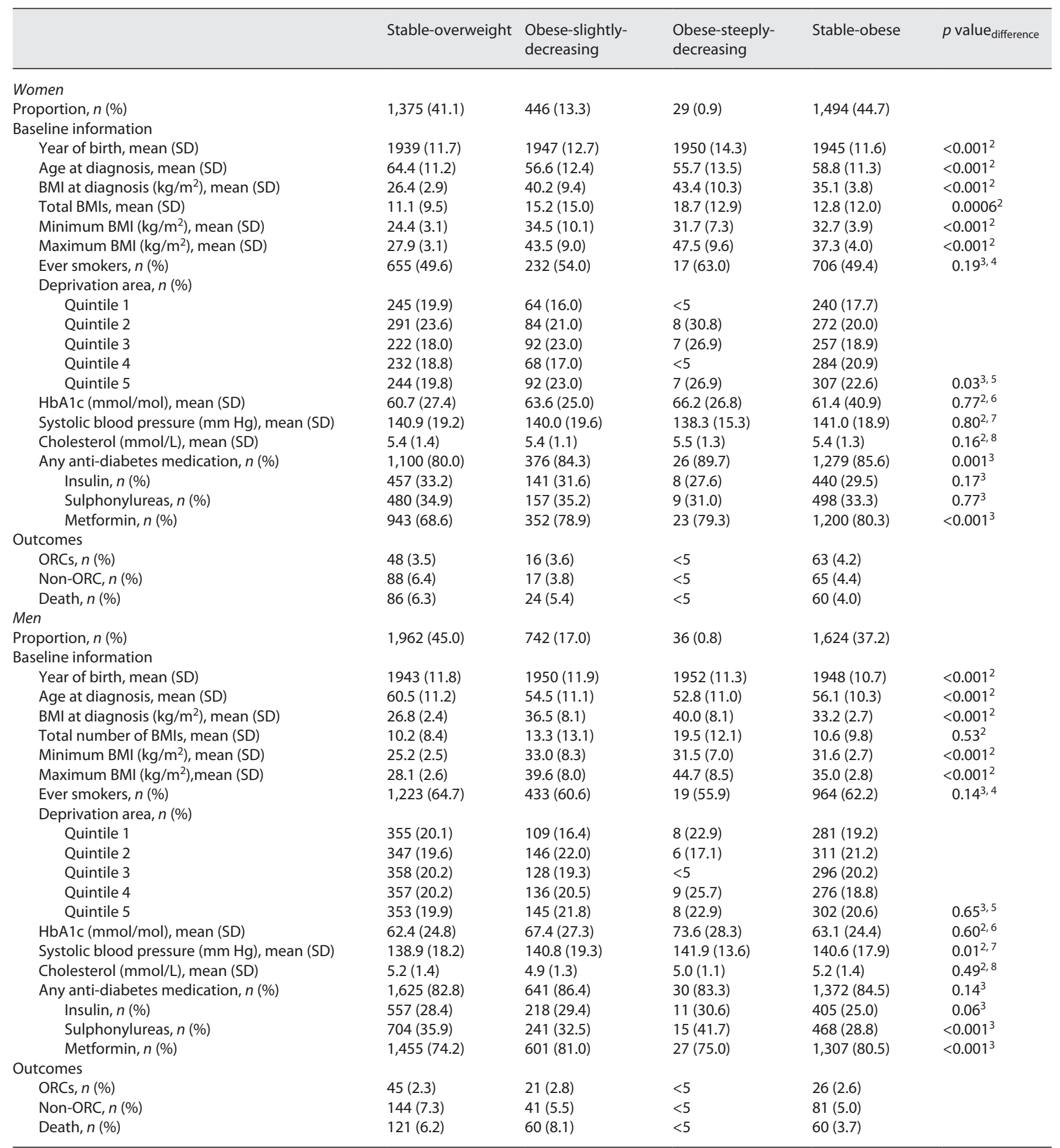

BMI, body mass index; HbA1c, glycated haemoglobin; ORC, obesity-related cancer; SD, standard deviation, SIR, Salford Integrated Record. ${ }^{1}$ Adjusting the $p$ value for multiple testing using the Bonferroni method, the significant level is $p=0.001 .{ }^{2}$ Difference between the classes were tested using ANOVA. ${ }^{3}$ Differences between the classes were tested using the $x^{2} .{ }^{4}$ Information on smoking was available for 3,209 women and 4,190 men. ${ }^{5}$ Information on deprivation area was available for 3,020 women and 3,935 men. ${ }^{6}$ Information on HbA1c was available for 2,783 women and 3,620 men. ${ }^{7}$ Information on blood pressure was available for 3,258 women and 4,271 men. ${ }^{8}$ Information on cholesterol was available for 3,306 women and 4,327 men. 


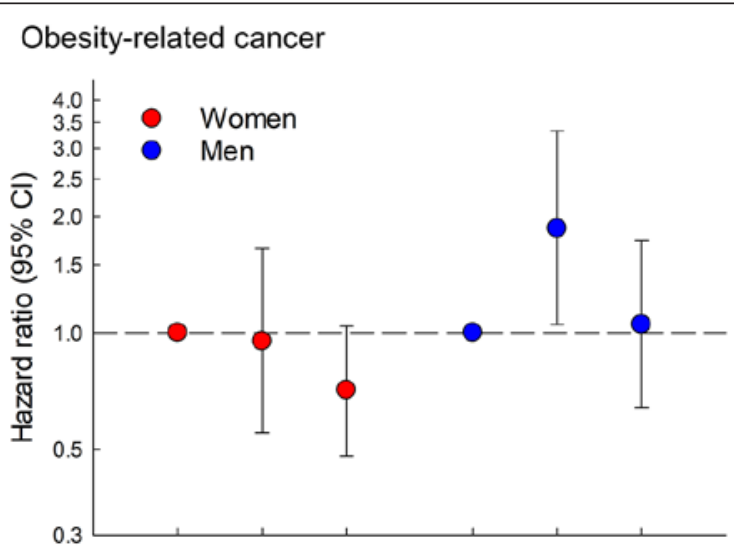

Non-obesity-related cancer

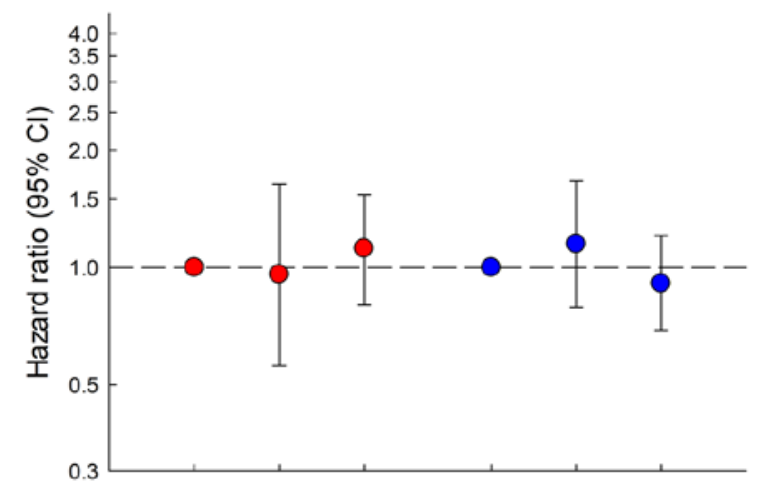

All-cause mortality

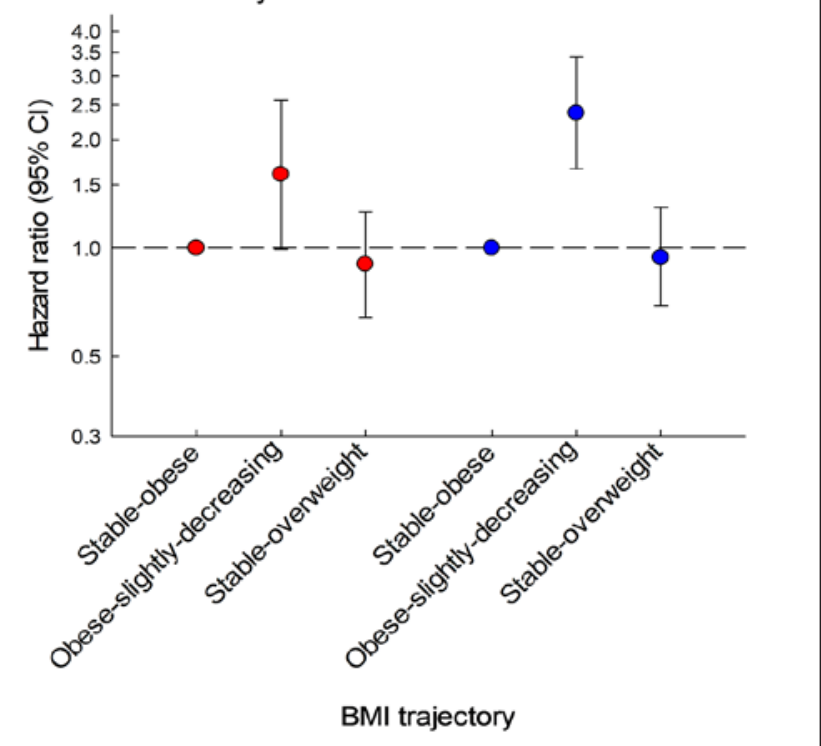

Fig. 3. Associations between BMI latent class trajectories and the risk of obesity-related, non-ORCs and all-cause mortality for men and women. Adjusted for age and calendar year of diagnosis. Due to few patients and a low number of cancer cases in the obesesteeply-decreasing class analyses on this class were not performed. BMI, body mass index; ORC, obesity-related cancer.

Latent BMI Classes, Diabetes, and Cancer portion of smokers, deprivation area, HbA1c, blood pressure, cholesterol, and insulin usage between the classes when accounting for multiple testing.

$\mathrm{BMI}$ at T2D diagnosis in the classes with decreasing BMI trajectories were covering the entire BMI spectrum. Thus, categorized according to the conventional WHO BMI categories these patients would not be identified as a separate class, but be allocated to different BMI categories (Fig. 2).

\section{Associations with ORCs}

During a median of 5.8 years (interquartile range: $2.9-9.0$ years) of observation time, 219 patients (127 women) were diagnosed with an ORC and 436 (170 women) with a nonORC among patients in the three analysed classes (Table 1). Compared with women in the stable-obese class, women in the stable-overweight class may have a lower risk of ORC even though it did not reach statistical significance (hazard ratio $[\mathrm{HR}]=0.71,95 \%$ confidence interval $[\mathrm{CI}]: 0.48-1.04$ ). In contrast, women in the obese-slightly-decreasing class had a similar risk of ORC as women in the stable-obese class; however, there were large CIs due to the relatively few cases (Fig. 3; online suppl. Table S3). Compared with men in the stable-obese class, no difference in risk of ORC was observed for men in the stable-overweight class, whereas men in the obese-slightly-decreasing class seem to have a higher risk of ORC (HR: 1.86, 95\% CI: 1.05-3.32).

\section{Associations with Non-ORCs}

Among women, no significant differences were observed between the classes in the association with nonORC (Fig. 3; online suppl. Table S3). Also, among men, no significant differences were observed in non-ORC risk between the classes. Excluding prostate cancer from the non-ORC cancers showed essentially similar results (online suppl. Table S4).

\section{Associations with All-Cause Mortality}

Compared to women in the stable-obese class, no difference in mortality was observed in the stable-overweight class, whereas a women in the obese-slightly-decreasing class may have a higher mortality although not statistically significant $(\mathrm{HR}=1.60,95 \% \mathrm{CI}: 0.99-2.58)$ (Fig. 3; online suppl. Table S3). Compared with men in the stable-obese class, no difference in mortality was observed for the stable-overweight class, whereas a higher mortality was observed in the obese-slightly-decreasing class (HR $=2.37,95 \% \mathrm{CI}: 1.66-3.39)$.

Adjustments for smoking and deprivation area showed essentially similar results (online suppl. Table S5). When 
the analyses were adjusted for BMI at T2D diagnosis, in general similar pattern in the results were observed. However, the probable inverse association for the stable-overweight class and ORC among women became non-significant (online suppl. Table S6).

No violations in the proportional hazard assumptions were observed (online suppl. Table S7). The analyses were repeated including only patients with a posterior probability $>70 \%$ and these showed essentially similar results (online suppl. Table S8).

\section{WHO BMI Categories}

When patients were categorized based on their BMI at T2D diagnosis using the WHO BMI categorization, women with obesity II or III had a higher risk of ORC $(\mathrm{HR}=1.61,95 \% \mathrm{CI}: 1.01-2.56)$ than women with obesity I (online suppl. Table S9). Women in all other categories had similar risk as the reference. Among men, no significant differences among the categories were observed in relation to ORC risk. Also, no differences in non-ORC risk were observed.

For all-cause mortality, we found no differences in the associations between the WHO BMI categories among women. However, in men both the under- and normal weight category (HR $=1.61,95 \% \mathrm{CI}: 1.10-2.36)$ and the obesity II and III category (HR $=1.52,95 \% \mathrm{CI}: 1.00-2.33$ ) may have higher mortality than men in the obesity I category.

\section{Discussion}

\section{Main Findings}

There were four key findings. Firstly, we identified four BMI latent class trajectories after T2D diagnosis, which were differently associated with ORCs. Secondly, the derived classes were different from conventional WHO BMI categories, and thus, LCTMs might add information to our understanding of weight changes. Thirdly, as expected, no associations were observed for nonORCs. Fourthly, we found a higher mortality in patients with an obese-slightly-decreasing BMI trajectory than a stable-obese BMI trajectory. A key study limitation was the lack of information on weight before T2D diagnosis and the limited follow-up time.

\section{Context of the Literature}

We used LCTM of repeated BMI values after T2D diagnosis, to simplify a heterogeneous population into more homogeneous classes [25]. LCTMs have been reported in general population studies with the following endpoints: all-cause mortality [27]; cancer incidence (multiple cancer types) [28]; gastro-oesophageal [29]; prostate [30]; and cancer mortality [29].

An American study including 1,064 patients with diabetes similarly to us identified four BMI trajectories using LCTM when examining associations with self-reported disability [31]. Their "stable overweight" and a "loss and regain obese" classes have similarities with our stableoverweight and stable-obese classes. In contrast to us, they also identified "stable normal" and "cumulating morbidly obese" classes, but not a class with a continuously decreasing BMI [31]. Supporting the decreasing BMI trajectories identified in our study, other studies examining changes in BMI, observed patients with a decrease in BMI post-T2D $[15,32,33]$.

Using hierarchical cluster methods a Swedish study among 12,112 patients with T2D, identified five different sub-types of T2D based on several parameters [34]. The sub-groups have parallels with the classes found in our study as $21.6 \%$ and $39.1 \%$ of the patients were categorised as having "mild obesity-related diabetes" or "mild agerelated diabetes," respectively. The former has similarities to our stable-obese class, while the latter is similar to our later age-onset stable-overweight class. Approximately $1 \%$ of men and women in our study were assigned to the obese-steeply-decreasing class, which may represent a specific group of patients. The significant weight loss observed in this class may be due bariatric surgeries which were indicated for patients with T2D and a BMI $>35 \mathrm{~kg} /$ $\mathrm{m}^{2}$ [35]. However, the frequency of bariatric surgery in the UK was low in the beginning of the study [36], and we cannot discount that the weight loss may be unintentionally and caused by reverse causation.

Contrary to what we expected, we did not observe a lower risk of ORC in the groups with decreasing BMI trajectories compared to the stable-obese class. However, the observed weight loss across the 10-year period in the two obese-slightly-decreasing classes was modest and may have been insufficient to affect cancer risk.

As cancers can take decades to develop from initial cell mutation to clinical manifestation, the BMI trajectory pre-T2D may impact on cancer risk [37], and previous studies have examined BMI trajectories prior to T2D diagnosis $[38,39]$. A moderate weight loss in mid-adult life may be too late to mitigate the negative effect of excess weight pre-T2D, possibly explaining the higher risk observed among men in the obese-slightly-decreasing class. The relatively few patients diagnosed with an ORC during follow-up may explain the lack of an association in women. 
The ORC are sex-specific [10] and the distribution of the cancer forms also differs between the sexes. This may explain the different associations observed and may also affect the presence of reverse causation between women and men. Additionally, women are generally heavier than men when diagnosed with T2D [40].

We found a higher mortality in the obese-slightly-decreasing class. While this class had a slightly decreasing trajectory especially among women, it remained at the highest BMI level. In a cohort study of 145,058 patients with T2D from the UK The Health Improvement Network database, the highest mortality was observed among patients with the highest BMI trajectory two years postT2D diagnosis among patients in the "Obese grade 3" class [19]. A meta-analysis including eight studies found, similar to us, that weight loss (based on two BMI values) among patients with T2D was associated with increased all-cause mortality, however, three included studies examining intentional weight loss reported no increased risk [41]. A study, including 173,246 Korean patients with newly diagnosed T2D, found a U-shaped relationship between two-year weight change after diagnosis and allcause mortality [42]. In our study, the mortality risk did not change during follow-up time ( $0-5$ vs. $>5$ years), indicating that the associations are likely not due to reverse causation. However, it is possible that patients in the obese-slightly-decreasing class had obesity for longer time compared to other patients and may suffer from a higher degree of multimorbidity, both of which may increase their overall mortality.

The differences in the associations between the conventional WHO BMI categories and the BMI trajectory classes, respectively, and ORC and mortality indicate that the BMI trajectories provide different information than BMI at diagnosis alone. Similarly, the Chord plots illustrate that the obese-slightly-decreasing class would not be identified using the traditional WHO BMI categories supporting the use of latent classes.

\section{Strengths and Weaknesses}

The study has several strengths. Firstly, there was a high density of repeated information on measured BMI. Secondly, we performed our LCTMs within a published framework to test model assumptions and justify class selection [25]. Thirdly, we linked our data with the cancer register to reduce cancer misclassification which could have occurred if only primary care records were used [43]. Fourthly, the data were from a stable population in Salford, with low rates of lost to follow-up. Fifthly, patient opt-out rates at the primary care level were $<0.2 \%[20]$.
There were study weaknesses. Firstly, the number of events during follow-up was limited. We addressed this by grouping cancers into ORCs and non-ORCs. Also, the date of death in the GP records may be delayed [44] but have improved over time and is unlikely to affect our results. Secondly, BMI determination in "real-world" data might be biased by opportunistic measurement. However, the trajectory of BMI changes is not predictive for subsequent BMI measurement [21]. Moreover, patients excluded due to no BMI $+/-1$ year of diagnosis with T2D were diagnosed with T2D earlier than patients included in the study and lack of BMI at diagnosis may be caused by procedural changes. Thirdly, within each class, some individuals had assignment probabilities $<70 \%$. However, excluding those individuals from analyses yielded similar results. Moreover, the heterogeneity in BMI within each class may have affected the results, but adjustment for BMI at diagnosis did not change the pattern of the results. Also, we did not identify a class with an increasing BMI trajectory. While we cannot preclude that there may be some patients with an increasing BMI trajectory, these are likely to be limited in number as the algorithm was unable to identify this pattern as a separate, robust trajectory. Fourthly, there is risk of residual confounding from measured and unmeasured factors. An example is the lack of information on physical activity, which might influence BMI trajectories and cancer risk. Finally, and importantly, we did not have information on weight before T2D diagnosis. Also, the limited time of follow-up and that no information was available on if the weight loss was intentional or not may have resulted in reverse causation. Moreover, patients diagnosed with T2D may be followed more closely than the general population increasing the risk of detection bias. However, the association did not differ when dividing the follow-time, indicating that this was not the case. The population in Salford is 95\% Caucasian, thus the patients with T2D in our study may not be representative of all patients with $\mathrm{T} 2 \mathrm{D}$ and the results of this study may not be generalizable to patients with T2D in the UK in general or to other European countries where the ethnic diversity may be larger.

\section{Clinical Implications}

It has recently been shown that the effect of weight loss in patients with T2D on CVD risk is inconsistent [18]. Based on the present findings, the association between BMI trajectories and cancer risk among patients with T2D is not clear either. Due to the limitations of the study, the results of the present study should not affect the current recommendation on weight loss in patients with $\mathrm{T} 2 \mathrm{D}$. 


\section{Unanswered Questions and Future Research}

Future studies examining the effect of weight changes in patients with T2D on the risk of ORC require a large sample size with a longer follow-up time including both women and men with repeated BMIs across the life course and information about intentional and unintentional weight loss. Future studies should consider quantification of visceral adiposity and fat in the liver, as these have strong correlations with insulin resistance, a potentially key biological mechanism for cancer development.

\section{Statement of Ethics}

Individual patient consent was not required; the data used contained anonymized patient data from routinely collected sources in the UK a network of GP practices and national cancer registry. All patients at the time of GP registration are given the option to withhold sharing their data. The study was approved by 11/ EM/0337 NREC committee East Midlands-Nottingham, UK. This study was performed in accordance with the Declaration of Helsinki.

\section{Conflict of Interest Statement}

A.G.R. has received lecture honoraria from Merck Serona and Janssen-Cilag, and independent research funding and lecture honoraria from Novo Nordisk and Sanofi Pasteur MPS, unrelated to this study. J.L.B. is an Associate Editor for Obesity Facts. All other authors have no conflicts of interest to declare.

\section{Funding Sources}

This paper presents independent research funded by the National Institute for Health Research (NIHR) under its Programme Grants for Applied Research (Grant reference No. RP-PG-0707-10031). A.G.R., C.W., and E.B. are supported by the Manchester NIHR Biomedical Research Centre (IS-BRC1215-20007). B.W.J. and J.L.B. were funded by Novo Nordisk Foundation (Grant No.: NNF17OC0028338) and the Dr. Sofus Carl Emil Friis and wife Olga Doris' Fund. The project was also supported by the World Cancer Research Fund.

\section{Author Contributions}

The followings are the authors' contribution: A.G.R. and E.B.: conceptualization; E.B.: data curation; B.W.J. and C.W.: formal analysis; A.G.R. and J.L.B.: funding acquisition; A.G.R., B.W.J., C.W., E.B., J.L.B., and N.G.: methodology; A.G.R., C.W., E.B., and N.G.: supervision; A.G.R. and B.W.J.: writing - original draft; C.W., E.B., J.L.B., and N.G.: writing - review and editing.

\section{Data Availability Statement}

The data used were anonymized records from individuals from the Salford Integrated Record. The ethical approval gained to use the data was prior to the general change in UK policy for data sharing (now overseen by the MHRA and NHS Digital). The approval for this data does not allow sharing or uploading of the individual records. Statistical code and clinical code sets can be provided on request.

\section{References}

1 Vazquez G, Duval S, Jacobs DR Jr, Silventoinen K. Comparison of body mass index, waist circumference, and waist/hip ratio in predicting incident diabetes: a meta-analysis. Epidemiol Rev. 2007;29:115-28.

2 Renehan A, Tyson M, Egger M, Heller RF, Zwahlen M. Body mass index and incidence of cancer: a systematic review and meta-analysis of prospective observational studies. Lancet. 2008;371(9612):569-78.

3 Lauby-Secretan B, Scoccianti C, Loomis D, Grosse Y, Bianchini F, Straif K. Body fatness and cancer - viewpoint of the IARC Working Group. N Engl J Med. 2016;375(8):794-8.

4 Giovannucci E, Harlan DM, Archer MC, Bergenstal RM, Gapstur SM, Habel LA, et al. Diabetes and cancer: a consensus report. Diabetes Care. 2010;33(7):1674-85.

5 Tsilidis KK, Kasimis JC, Lopez DS, Ntzani EE, Ioannidis JP. Type 2 diabetes and cancer: umbrella review of meta-analyses of observational studies. BMJ. 2015;350:g7607.

6 Ohkuma T, Peters SAE, Woodward M. Sex differences in the association between diabetes and cancer: a systematic review and meta- analysis of 121 cohorts including 20 million individuals and one million events. Diabetologia. 2018;61(10):2140-54.

7 Renehan A, Smith U, Kirkman MS. Linking diabetes and cancer: a consensus on its complexity. Lancet. 2010;375(9733):2201-2.

8 Badrick E, Sperrin M, Buchan IE, Renehan AG. Obesity paradox and mortality in adults with and without incident type 2 diabetes: a matched population-level cohort study. BMJ Open Diabetes Res Care. 2017;5(1): e000369.

9 Drake I, Gullberg B, Sonestedt E, Stocks T, Bjartell A, Wirfalt E, et al. Type 2 diabetes, adiposity and cancer morbidity and mortality risk taking into account competing risk of noncancer deaths in a prospective cohort setting. Int J Cancer. 2017;141(6):1170-80.

10 Hendriks SH, Schrijnders D, van Hateren KJ, Groenier KH, Siesling S, Maas A, et al. Association between body mass index and obesityrelated cancer risk in men and women with type 2 diabetes in primary care in the Netherlands: a cohort study (ZODIAC-56). BMJ Open. 2018;8(1):e018859.
11 Miao Jonasson J, Cederholm J, Gudbjornsdottir S. Excess body weight and cancer risk in patients with type 2 diabetes who were registered in Swedish National Diabetes Register - register-based cohort study in Sweden. PLoS One. 2014;9(9):e105868.

12 Yamamoto-Honda R, Takahashi Y, Yoshida Y, Kwazu S, Iwamoto Y, Kajio H, et al. Body mass index and the risk of cancer incidence in patients with type 2 diabetes in Japan: results from the National Center Diabetes Database. J Diabetes Investig. 2016;7(6):90814.

13 Franz MJ, Boucher JL, Rutten-Ramos S, VanWormer JJ. Lifestyle weight-loss intervention outcomes in overweight and obese adults with type 2 diabetes: a systematic review and metaanalysis of randomized clinical trials. J Acad Nutr Diet. 2015;115(9):1447-63.

14 Lean ME, Leslie WS, Barnes AC, Brosnahan N, Thom G, McCombie L, et al. Primary careled weight management for remission of type 2 diabetes (DiRECT): an open-label, clusterrandomised trial. Lancet. 2018;391(10120): 541-51. 
15 Aucott LS, Philip S, Avenell A, Afolabi E, Sat$\operatorname{tar}$ N, Wild S. Patterns of weight change after the diagnosis of type 2 diabetes in Scotland and their relationship with glycaemic control, mortality and cardiovascular outcomes: a retrospective cohort study. BMJ Open. 2016; 6(7):e010836.

16 Bodegard J, Sundström J, Svennblad B, Östgren CJ, Nilsson PM, Johansson G. Changes in body mass index following newly diagnosed type 2 diabetes and risk of cardiovascular mortality: a cohort study of 8486 primarycare patients. Diabetes Metab. 2013;39(4): 306-13.

17 Gregg EW, Jakicic JM, Blackburn G, Bloomquist P, Bray GA, Clark JM, et al. Association of the magnitude of weight loss and changes in physical fitness with long-term cardiovascular disease outcomes in overweight or obese people with type 2 diabetes: a post-hoc analysis of the Look AHEAD randomised clinical trial. Lancet Diabetes Endocrinol. 2016;4(11):913-21.

18 Strelitz J, Ahern AL, Long GH, Hare MJL, Irving $\mathrm{G}$, Boothby $\mathrm{CE}$, et al. Moderate weight change following diabetes diagnosis and 10 year incidence of cardiovascular disease and mortality. Diabetologia. 2019;62(8):1391402.

19 Owusu ESA, Samanta M, Shaw JE, Majeed A, Khunti K, Paul SK. Weight loss and mortality risk in patients with different adiposity at diagnosis of type 2 diabetes: a longitudinal cohort study. Nutr Diabetes. 2018;8(1):37.

20 New JP, Leather D, Bakerly ND, McCrae J, Gibson JM. Putting patients in control of data from electronic health records. BMJ. 2018; 360:j5554.

21 Sperrin M, Petherick E, Badrick E. Informative observation in health data: association of past level and trend with time to next measurement. Stud Health Technol Inform. 2017; 235:261-5.

22 de Lusignan S, Khunti K, Belsey J, Hattersley A, van Vlymen J, Gallagher H, et al. A method of identifying and correcting miscoding, misclassification and misdiagnosis in diabetes: a pilot and validation study of routinely collected data. Diabet Med. 2010;27(2):203-9.

23 Sacks DB. Measuring and reporting hemoglobin Alc. Clin Biochem. 2012;45(13-14): 1046-7.
24 McLennan D, Barnes H, Noble M, Dibben C. The English indices of deprivation 2010 for the Department for Communities and Local Government. 2011. Available from: www.gov. uk/government/uploads/system/uploads/attachment_data/file/6320/1870718.pdf Accessed 2020 Mar 18.

25 Lennon H, Kelly S, Sperrin M, Buchan I, Cross AJ, Leitzmann M, et al. Framework to construct and interpret latent class trajectory modelling. BMJ Open. 2018;8(7):e020683.

26 World Cancer Research Fund/American Institute for Cancer Research. Diet, Nutrition, Physical Activity and Cancer: a Global Perspective. Continous Update Project Expert Report. 2018.

27 Song $\mathrm{M}, \mathrm{Hu} \mathrm{FB}, \mathrm{Wu} \mathrm{K}$, Must A, Chan AT, Willett WC, et al. Trajectory of body shape in early and middle life and all cause and cause specific mortality: results from two prospective US cohort studies. BMJ. 2016;353:i2195.

28 Song M, Willett WC, Hu FB, Spiegelman D, Must A, Wu K, et al. Trajectory of body shape across the lifespan and cancer risk. Int J Cancer. 2016;138(10):2383-95.

29 Petrick JL, Kelly SP, Liao LM, Freedman ND, Graubard BI, Cook MB. Body weight trajectories and risk of oesophageal and gastric cardia adenocarcinomas: a pooled analysis of NIH-AARP and PLCO Studies. Br J Cancer. 2017;116(7):951-9.

30 Kelly SP, Graubard BI, Andreotti G, Younes N, Cleary SD, Cook MB. Prediagnostic body mass index trajectories in relation to prostate cancer incidence and mortality in the PLCO cancer screening trial. J Natl Cancer Inst. 2017;109(3):djw225.

31 Chiu CJ, Wray LA, Lu FH, Beverly EA. BMI change patterns and disability development of middle-aged adults with diabetes: a dual trajectory modeling approach. J Gen Intern Med. 2013;28(9):1150-6.

32 Looker HC, Knowler WC, Hanson RL. Changes in BMI and weight before and after the development of type 2 diabetes. Diabetes Care. 2001;24(11):1917-22.

33 de Fine Olivarius N, Andreasen AH, Siersma V, Richelsen B, Beck-Nielsen H. Changes in patient weight and the impact of antidiabetic therapy during the first 5 years after diagnosis of diabetes mellitus. Diabetologia. 2006;49(9): 2058-67.
34 Ahlqvist E, Storm P, Karajamaki A, Martinell M, Dorkhan M, Carlsson A, et al. Novel subgroups of adult-onset diabetes and their association with outcomes: a data-driven cluster analysis of six variables. Lancet Diabetes Endocrinol. 2018;6(5):361-9.

35 O'Connor D. Anaesthesia for bariatric surgery guide. 2019. Northern Care Alliance. Avaliable on: www.srft.nhs.uk/EasysiteWeb/ getresource.axd? AssetID=32212\& type =full $\&$ servicetype=Inline Accessed 2020 Mar 18.

36 Booth HP, Khan O, Fildes A, Prevost AT, Reddy M, Charlton J, et al. Changing epidemiology of bariatric surgery in the UK: cohort study using primary care electronic health records. Obes Surg. 2016;26(8):1900-5.

37 Meza R, Jeon J, Renehan AG, Luebeck EG. Colorectal cancer incidence trends in the United States and United Kingdom: evidence of right- to left-sided biological gradients with implications for screening. Cancer Res. 2010; 70(13):5419-29.

38 Vistisen D, Witte DR, Tabak AG, Herder C, Brunner EJ, Kivimaki M, et al. Patterns of obesity development before the diagnosis of type 2 diabetes: the Whitehall II cohort study. PLoS Med. 2014;11(2):e1001602.

39 Kuwahara K, Honda T, Nakagawa T, Yamamoto S, Hayashi T, Mizoue T. Body mass index trajectory patterns and changes in visceral fat and glucose metabolism before the onset of type 2 diabetes. Sci Rep. 2017;7:43521.

40 Logue J, Walker JJ, Colhoun HM, Leese GP, Lindsay RS, McKnight JA, et al. Do men develop type 2 diabetes at lower body mass indices than women? Diabetologia. 2011;54(12):3003-6.

41 Chen Y, Yang X, Wang J, Li Y, Ying D, Yuan $\mathrm{H}$. Weight loss increases all-cause mortality in overweight or obese patients with diabetes: a meta-analysis. Medicine. 2018;97(35):e12075.

42 Kim MK, Han K, Koh ES, Kim ES, Lee MK, Nam GE, et al. Weight change and mortality and cardiovascular outcomes in patients with new-onset diabetes mellitus: a nationwide $\mathrm{Co}^{-}$ hort study. Cardiovasc Diabetol. 2019;18(1):36.

43 Badrick E, Renehan I, Renehan AG. Linkage of the UK clinical practice research datalink with the national cancer registry. Eur J Epidemiol. 2019;34(1):101-2.

44 Harshfield A, Abel GA, Barclay S, Payne RA. Do GPs accurately record date of death? A UK observational analysis. BMJ Support Palliat Care. 2020;10(3):e24. 